\title{
Time-Correlated Single Photon Counting Spectroscopy: Principles and Applications to Single-Filament Discharges
}

\author{
Tomáš Hoder ${ }^{1}$, Mirko Černák \\ Department of Physical Electronics, Faculty of Science, Masaryk University \\ Kotlarska 2, 61137 Brno, Czech Republic \\ E-mail: hoderaphysics.muni.Cz \\ Hans Höft, Torsten Gerling, Ronny Brandenburg \\ Leibniz-Institute for Plasma Science and Technology, INP Greifswald \\ Felix-Hausdorff-Str. 2, 17489 Greifswald, Germany
}

Time-correlated single photon counting (TC-SPC) is a powerful optical emission spectroscopy technique for investigation of repetitive discharge events. It offers a high temporal resolution, high dynamic range and possibility to trigger on stochastically appearing plasmas. TC-SPC technique is found to be very effective in investigation of the fundamental breakdown phenomena where all three above mentioned advances are of high significance. Here, we present briefly the principles and basics of this technique, reveal the history of its application in discharge physics and introduce several examples of its application made recently. The contribution refers to more detailed expertise in main articles on the topic.

First EPS Conference on Plasma Diagnostics - $1^{\text {st }}$ ECPD

14-17 April 2015,

Villa Mondragone, Frascati (Rome) Italy

${ }^{1}$ Speaker 


\section{Introduction}

Low temperature atmospheric pressure micro-plasmas are widely used in different applications such as ozone generation, air or water purification, polymer surface treatment, combustion or medicine etc. [1-3]. At atmospheric pressure, these plasmas take usually form of thin filaments of sub-mm dimensions. Typically, they are ruled by two distinct gas discharge mechanisms. One of them is dominant at lower electric fields and leads to the free charge accumulation and usually can be described by simple Townsend theory [4] where the secondary gamma processes have mostly not enough time to fully develop. The second mechanism is the result of this charge accumulation and is called streamer [4-7]. Under given conditions on sub$\mathrm{mm}$ spatial scales, the electric fields under which the gas ionization happens differ up to few orders of magnitude for these two mechanisms. As a consequence, the magnitude of the emitted light or the velocities of the ionizing front development vary on relatively large intervals. Therefore, to record the complete development of the filamentary plasma from its slow weaklyemitting beginning up to its ultra-fast high-field gas-breakdown is true challenge for experimental plasma diagnostics.

In order to follow all above mentioned processes and stages of the atmospheric pressure filamentary discharge we utilized and applied the time-correlated single photon counting technique (also called Cross-Correlation Spectroscopy - CCS [8]) which offers high-temporal resolution, high dynamic range and possibility to trigger on stochastically appearing plasmas. In this contribution, we present the principle of this technique, reveal the history of its application in discharge physics and introduce several examples of its application recently made.

\section{Principle and realization}

As it was briefly written by Kozlov in [8]: "The main idea of the method of CCS is to replace a direct measurement of the single pulse of luminosity from a repetitive light pulse emitter by a statistically averaged determination of the correlation function between two optical signals, both originating from the same source. The first of these signals (the so-called 'synchronizing signal') is used to define a relative time scale, the second one (the 'main signal') has to be detected with a probability at least one order of magnitude lower than that of the synchronizing signal. The measured quantity is actually a time delay between these two signals, and the recorded quantity is a probability density function for the evolution of the light pulse intensity. If the repetitive light pulses reproduce each other sufficiently exactly, and if the synchronizing signal detection is adjusted in such a way as to occur always at the same moment that a single light pulse evolves, then the recorded probability density function is proportional to the light intensity I ( $t$ ) of the source under consideration." The technique has an accumulative nature. The measured temporal correlations, i.e. times measured between the 'main' and the 'sync' signal, are added as digital units/counts to the memory boxes with corresponding time indexes. Finally, if the sample/micro-discharge is scanned spatially, for each position one obtains a histogram representing the emission development in time. Further details for TC-SPC technigue can be also found in following scientific contributions $[9,10,11]$. The schematic presentation of the TC-SPC concept is shown in Figure 1a). 
a)

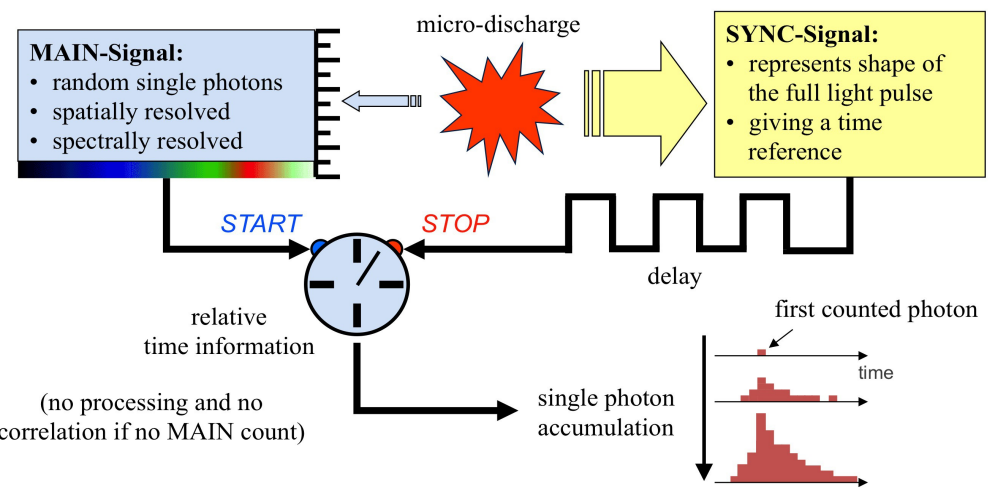

b)

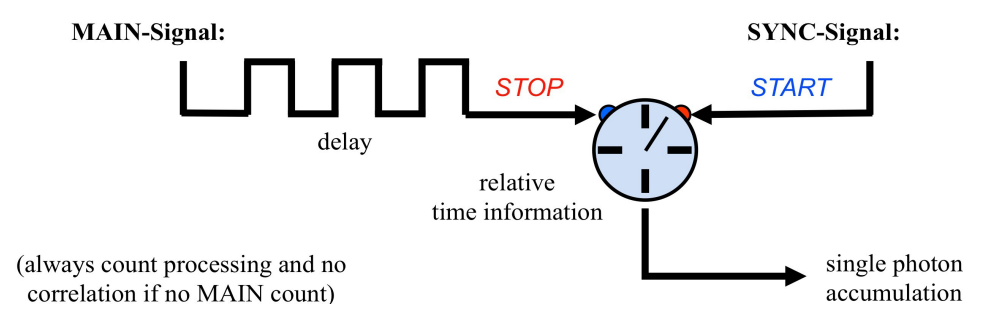

Figure 1: The principle of the TC-SPC technique in its reverse start-stop modification is shown in part (a). This arrangement was also usually used in gas-discharge applications. In part (b) the direct startstop configuration is presented.

In other words, the TC-SPC technique measurement happens in following way. First, one has a light signal with certain information (selected wavelength or spatial coordinate) coming from low-level, high-repetition-rate source (in our case a micro-discharge at least $\mathrm{kHz}$ frequency). Using a photomultiplier (PMT) in a single photon counting mode directed toward the source one gets a train of single-photon pulses. The pulse density signal, rather than the signal amplitude, provides the measure of the light intensity at the input of the detector. The pulses are detected/resolved by a discriminator. The output pulses of the discriminator must be counted into a large number of memory channels according to their time in the signal period. Which means, when a photon is detected in TC-SPC experiment, the time of the corresponding detector pulse in the signal period is measured with respect to the reference signal. The signal period is determined by the routing procedure and the synchronization/reference signal is, for example, the total emission pulse of the discharge itself. The events are collected in a memory adding a "1" in a memory location/channel with an address proportional to the detection time [10]. Obviously, TC-SPC is a digital technique.

In general in TC-SPC technique, the synchronization does not have to be the microdischarge light emission itself, but also the discharge current pulse, TTL pulse of power supply or other triggering/characteristic event connected to the sample under investigation. In the subnanosecond ionization discharge physics however, these other methods can insert additional issues in to the system and could negatively affect the final result more than the straight forward optical synchronization. 


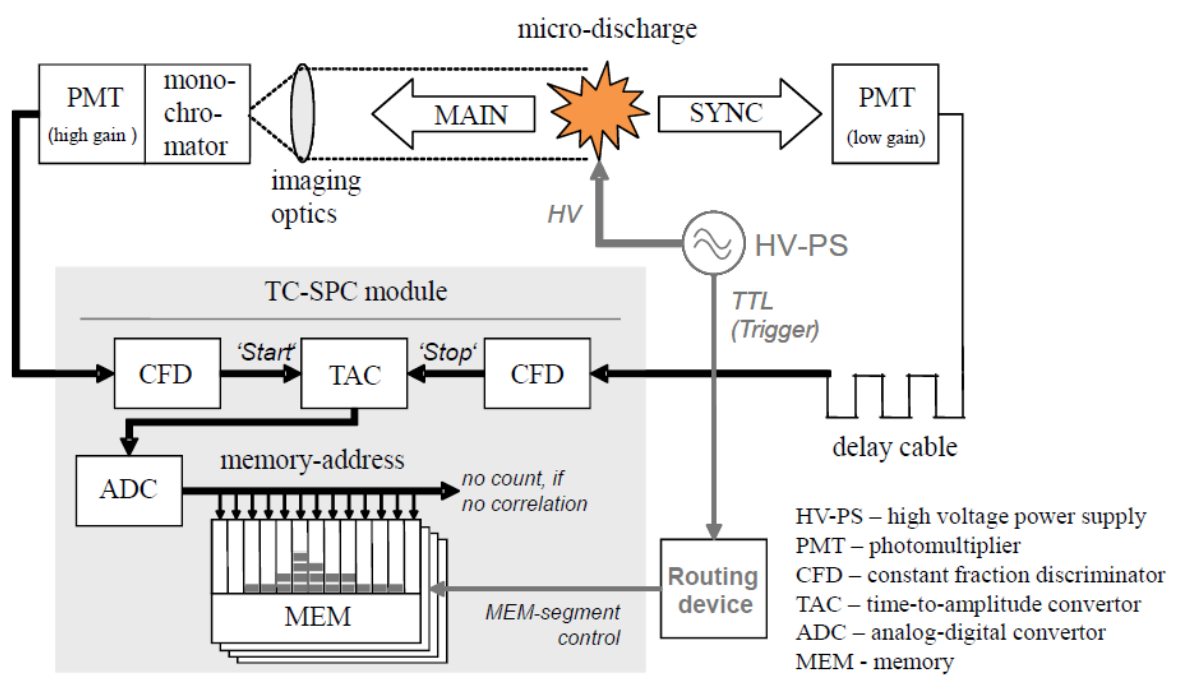

Figure 2: The technical realization of the TC-SPC technique in its reverse start-stop modification.

The technical realization of the TC-SPC setup is shown in Fig.2. It consists of two optoelectronic paths, one for the main and one for the synchronization signal. The main signal is spatially selected from the discharge using imaging optics, spectrally resolved by its passage through the monochromator and detected as a single photon via high gain photomultiplier (PMT). The single photon electrical pulse from PMT goes further to the constant fraction discriminator (CFD) which is utilizing the constant fraction triggering approach (far more precise than the leading edge triggering method, see [10]). This temporally well defined signal triggers the time measurement (linear voltage increase) in the time-to-amplitude converter unit (TAC). This is stopped by the arrival of the synchronization signal - neither spatially, nor spectrally resolved pulse representing the time reference. The synchronization signal is recorded by the low gain PMT and in reversed start-stop arrangement delayed by the prolonged $\mathrm{BNC} / \mathrm{SMA}$ cable.

As already announced, there are two photon counting correlation arrangements. The so called direct start-stop and reversed start-stop correlation, which are both schematically shown in Fig.1. Originally, the system with direct start-stop is used in lower frequency laser excitation of fluorescence imaging [10]. The TAC is started every time the synchronization signal is detected (in fluorescence spectroscopy with every excitation pulse) and stopped with the statistical detection of main signal (which must be one or two orders of magnitude less frequent, see furher). For frequencies in tens of $\mathrm{MHz}$ and higher it is no more applicable. The TAC must be reset each 10 or $20 \mathrm{~ns}$, while measuring some rare detection events between the reset pulses. Thus high-repetition systems work in the reversed start stop configuration: the TAC is started when a photon is detected and stopped with the next reference pulse from the light source. Consequently, the TAC has to work only at the lower rate of the photon detection events (main signal pulses), not at the much higher rate of the excitation pulses (here in our case the synchronization pulses) [10].

If, following one micro-discharge pulse in direct start-stop arrangement, the registering of a coincident event in channel $i$ corresponds to an average number of $n_{i}$ photons reaching the 
photocathode of the "stop" PMT and liberating an average number of $x_{i}$ photoelectrons, then $x_{i}$ $=n_{i} \cdot q$, where $q$ is the photocathode quantum efficiency. If there are a large number of excitation pulses for every count registered in channel $i$, then the probability $P_{x}(i)$ of liberating other than $\mathrm{x}_{i}$ photoelectrons is given by the Poisson distribution,

$$
P_{x}(i)=\frac{x_{i}^{x} \mathrm{e}^{-x_{i}}}{x !} \text {, with } \sum_{x=0}^{\infty} P_{x}(i)=1,
$$

Therefore, the probability per micro-discharge pulse of at least one photo-electron detected is given by

$$
P_{x \geqslant 1}(i)=1-P_{0}(i)=1-\mathrm{e}^{-x_{i}}=1-\left(1-x_{i}+\frac{x_{i}^{2}}{2}+\ldots\right), \text { which is } \quad P_{x \geqslant 1}(i) \simeq x_{i} \simeq n_{i} q
$$

for very low light levels (i.e. if $x_{i}>>x_{i}^{2} / 2$, usually it means that the synchronization signal rate is hundred-times higher than the main signal one). Under these conditions, the probability of detecting a coincident event in channel $i$ is proportional to the discharge intensity at a time $t$. This is the general condition which is essential for accurate single-photon counting measurements [11].

For the application of the TC-SPC method to the discharge/plasma research these two mentioned start-stop configurations have following importance. In the case of the direct startstop arrangement, the resulting recording/histogram starts at the time of synchronization signal arrival or a bit earlier (caused by the delay on the main signal line, see Fig.1b)), so the detection of the emission after the breakdown is easily practicable. This is used for study of the discharge afterglow, relaxation processes or to investigate the subsequent appearance of the next breakdown at the same spatial coordinate [12]. In the case of reversed start-stop arrangement, the emission prior to the breakdown event can be studied preferentially. This covers mostly the pre-breakdown phenomena connected to the slow charge accumulation prior to the breakdown $[7,13]$. For more complex review of the physical phenomena participating in the microdischarge development and studied not only via optical emission spectroscopy the reader is referred to following review papers [14-16].

The time intervals preceding or following the trigger pulse of the synchronization signal can go from picoseconds up to microseconds in both start-stop arrangements. It depends of course on the time scale definition of the memory blocks (as shown in Fig.2) and on the quality of the "delay cable" utilization. Recently, modern TC-SPC devices (e.g. here [10]) has both (the direct as well as the reversed) start-stop configuration settings, switchable as needed.

\section{Brief history}

The TC-SPC principles were used for the first time for fluorescence measurements in pulse fluorometry by late 50 's. Soon after, the applications of this method spread from photophysics, photo-chemistry to photo-biology [9]. In Japan in late 70's, Ikuta and Kondo applied the TC-SPC technique probably for the first time in gas discharge physics [17]. They investigated streamer discharges in positive as well as negative corona [17]. In their work, the spectral bands with 0-0 vibrational transition of second positive (SPS, at $337 \mathrm{~nm}$ ) and first negative (FNS, at $391 \mathrm{~nm}$ ) systems of molecular nitrogen were chosen for detection. This 
approach was motivated by the results of Gallimberti [18] who used these spectral bands to approach the electron energy in the discharge. Similarly, using the SPS emission the Trichel pulse discharge in low pressure negative corona was investigated by Gravendeel [19]. Using the TC-SPC technique, Gravendeel reached the temporal resolution of $0.7 \mathrm{~ns}$ in late 80 's. At the end of 90 's, the understanding of streamer discharges using optical emission spectroscopy was improved significantly by Matveev, Djakov, Shcherbakov, Kozlov and their co-workers [8,2023]. While Matveev and Djakov used a theoretical approach, the experimental approach used by Shcherbakov and Kozlov was based on TC-SPC technique principles. Kozlov, Wagner and coworkers successfully applied the TC-SPC technique to determine the spatio-temporal development of the electric field in barrier discharges in atmospheric pressure air with subnanosecond and sub-mm resolution [8]. They used the fact that the intensity ratio of FNS to SPS light emissions is strongly dependent on the electric field [24]. Moreover, from such a result they were able to estimate the relative electron density and local ozone production as well [8]. Later in 2007, Shcherbakov developed a complex approach to analyze the streamer discharge thoroughly using TC-SPC-similar device based also on cross-correlation of two signals coming from the same streamer discharge $[25,26]$.

\section{Recent examples of TC-SPC application}

Although the self-pulsing mode of Trichel pulse negative corona is known for a very long time [27], its precise understanding, accompanied with high-resolution experimental determination of e.g. electric field development, was still missing. We applied the TC-SPC technique to record the FNS and SPS emissions with resolution of tens of micrometers and tens of picoseconds [7]. Using the calibrated FNS/SPS ratio dependency on the electric field $[8,18,24]$ we determined the electric field development with above mentioned resolution. In Fig.3., the setup of the Trichel pulse experiment is shown. A single table setup was arranged including the high-resolution and high dynamic range detection device together with the calibration path with non-self-sustaining Townsend discharge. The experimental conditions for negative corona in this case resulted in Trichel pulsing mode with frequency of approx. $200 \mathrm{kHz}$ and single pulse amplitude of $4 \mathrm{~mA}$ [7]. As a result of the well resolved emission recording and determined electric field development we succeeded to recognize the propagation of both negative and positive streamers within this nanosecond breakdown. The maximal value of the reduced electric field for the negative streamer approached to $500 \mathrm{Td}$ while for the positive streamer it was nearly two times higher [7]. This was for the first time such a high-electric field ionization was detected within the Trichel pulse discharge. Based on this result we proposed a unified breakdown theory stating that the formation of a primary cathode-directed streamer occurs always in any streamer-initiated breakdown and pre-breakdown phenomena associated with cathode spot formation [7]. 


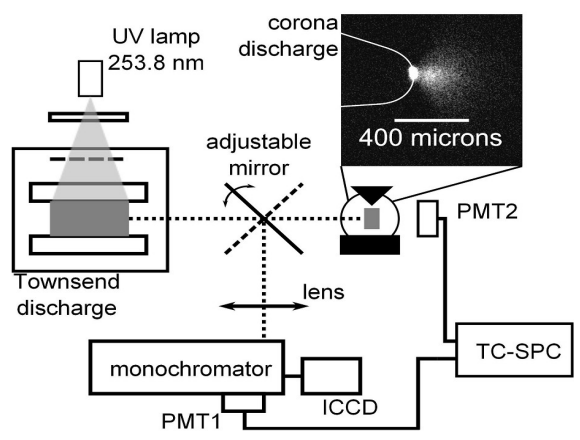

Figure 3: Experimental setup for the Trichel pulse analysis [7] consisting of Townsend discharge for $\mathrm{FNS} / \mathrm{SPS}=\mathrm{f}(\mathrm{E} / \mathrm{N})$ calibration $(\mathrm{E} / \mathrm{N}$ denotes reduced electric field) in the left upper part and of negative corona discharge under investigation (right part of the figure). Below, the TC-SPC setup is schematically presented.

For the first time, the fast breakdown in pulsed barrier discharge was recorded simultaneously by two different high-resolution methods in [14]. Apart from the TC-SPC recordings (see Fig.4), the streak camera image via far-field microscope was taken. After the comparison, both obtained results agreed synergistically [14]. The TC-SPC measurement showed higher sensitivity, however the streak camera with slightly lower dynamic range collected the data much faster. Pre-breakdown phenomena in sine voltage waveform driven barrier discharges was investigated in [13]. For more information on sine waveform driven volume, surface or coplanar barrier discharge see please references [13,28-33] (discharges driven in air and air-like mixtures and pure argon or argon-ammonia mixture were investigated). For pulsed driven barrier and corona discharges development recorded by streak or TC-SPC devices see $[28,29,34]$ and references therein.

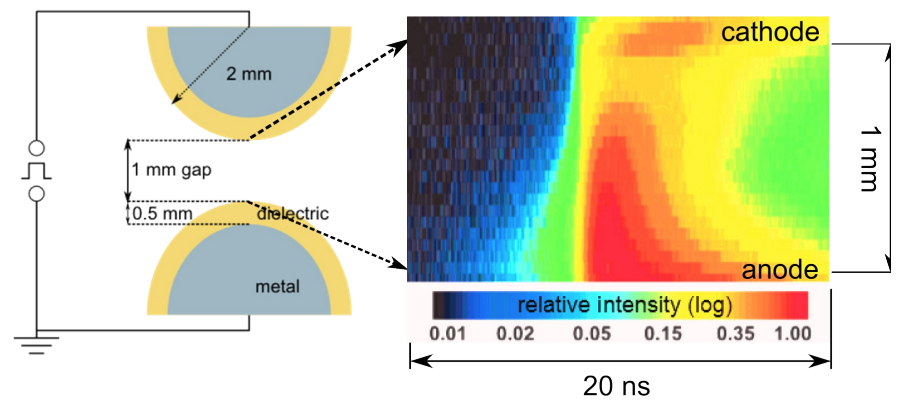

Figure 4: The development of the SPS emission in pulsed DBD recorded by the TC-SPC technique [14]. The signal intensity goes in a logarithmic scale from low values depicted by blue color, to higher in yellow and the highest in red. The propagation of the positive streamer (in blue contour: from the left bottom corner towards the cathode) and creation of the transient glow discharge (in time around of $10 \mathrm{~ns}$ of development) is visible.

Transient spark discharge was studied in details by TC-SPC in $[35,36]$ for broad interval of wavelengths (from UV for molecular nitrogen band spectra to near IR emission for argon and oxygen atomic lines). The challenge was to detect at least tens of photons for given intervals of the discharge development emission as the repetition frequency was at the lower limit of the 
method possibilities, i.e. around $1 \mathrm{kHz}$. The detected signal was already affected by statistical artifacts (after-pulsing [10] etc.). However, proper analysis was made for correct interpretation of the recordings.

\section{Summary and conclusion}

TC-SPC is unique diagnostic technique, which enables a deeper insight into the elementary processes before and during the gas-breakdown in filamentary plasmas. In this invited contribution the principles of this technique were presented together with brief overview of previous and recent highlights of application of this technique to barrier, corona or transient spark discharges. Additionally, relevant references are listed for further, more detailed, reading.

\section{Acknowledgement}

Authors are grateful to K.V.Kozlov and H.-E. Wagner for fruitful discussions. This research was supported by the project of Deutsche Forschungsgemeinschaft, Collaborative Research Centre 24 (TRR24) Fundamentals of Complex plasmas, the Czech Science Foundation research project 15-04023S and by the project CZ.1.05/2.1.00/03.0086 funded by European Regional Development Fund and project LO1411 (NPU I) funded by Ministry of Education, Youth and Sports of Czech Republic.

\section{References}

[1] K. H. Becker, U. Kogelschatz, K. H. Schoenbach and R. J. Barker eds., Non-equilibrium air plasmas at atmospheric pressure, Bristol and Philadelphia: IOP, 2004

[2] V. L. Parvulescu, M. Magureanu, P. Lukeš eds., Plasma Chemistry and Catalysis in Gases and Liquids, Wiley-VCH: Weinheim, Germany, 2012

[3] Th. von Woedtke, S. Reuter, K. Masur, K.-D. Weltmann, Plasmas for medicine, Physics Reports 530 (2013) 291

[4] Yu. P. Raizer Gas discharge physics, Springer-Verlag: Berlin Heidelberg, Germany, 1991

[5] H. Raether, Die Entwicklung der Elektronenlawine in den Funkenkanal, Z. Phys. 112 (1939) 464

[6] R. V. Hodges, R. N. Varney and J. F. Riley, Probability of electrical breakdown: Evidence for a transition between the Townsend and streamer breakdown mechanisms, Phys. Rev. A 31 (1985) 2610

[7] T. Hoder, M. Cernak, J. Paillol, D. Loffhagen and R. Brandenburg, High-resolution measurements of the electric field at the streamer arrival to the cathode: a unification of the streamer-initiated gas-breakdown mechanism, Phys. Rev. E, 86 (2012) 055401(R)

[8] K. V. Kozlov, H.-E. Wagner, R. Brandenburg and P. Michel, Spatio-temporally resolved spectroscopic diagnostics of the barrier discharge in air at atmospheric pressure, J. Phys. D: Appl. Phys. 34 (2001) 3164

[9] W. R. Ware, Technique of pulse fluorometry Time-Resolved Fluorescence Spectroscopy in Biochemistry and Biology (NATO ASI Series A: Life Sciences) 69, eds. R B Cundall and R E Dale, New York: Plenum, pp 23-57, 1983

[10] W. Becker Advanced Time-Correlated Single Photon Counting Techniques, Berlin: Springer, 2005 
[11] J. R. Lakowicz Topics in fluorescence spectroscopy, New York: Kluwer Academic Publishers, 2002

[12] R. Brandenburg, H.-E. Wagner, A. M. Morozov and K. V. Kozlov, Axial and radial development of microdischarges of barrier discharges in N2/O2 mixtures at atmospheric pressure, J. Phys. D: Appl. Phys. 38 (2005) 1649

[13] T. Hoder, R. Brandenburg, R. Basner, K.-D. Weltmann, K. V. Kozlov and H.-E. Wagner, $A$ comparative study of three different types of barrier discharges in air at atmospheric pressure by cross-correlation spectroscopy, J. Phys. D: Appl. Phys. 43 (2010) 124009

[14] R. Brandenburg, M. Bogaczyk, H. Hoeft, S. Nemschokmichal, R. Tschiersch, M. Kettlitz, L. Stollenwerk, T. Hoder, R. Wild, K.-D. Weltmann, J. Meichsner and H.-E. Wagner, Novel insights into the development of barrier discharges by advanced volume and surface diagnostics, J. Phys. D: Appl. Phys. 46 (2013) 464015

[15] P. Bruggeman and R. Brandenburg, Atmospheric pressure discharge filaments and microplasmas: physics, chemistry and diagnostics, J. Phys. D: Appl. Phys. 46 (2013) 464001

[16] M. Simek, Optical diagnostics of streamer discharges in atmospheric gases, J. Phys. D: Appl. Phys. 47 (2014) 463001

[17] N. Ikuta and K. Kondo, A spectroscopic study of positive and negative coronas in $\mathrm{N}_{2} \mathrm{O}_{2}$ mixture, in IEE Proceedings of 4th International Conference on Gas Discharges, 7-10 September 1976.

[18] I. Gallimberti, J. K. Hepworth, and R. C. Klewe, Spectroscopic investigation of impulse corona discharges J. Phys. D: Appl. Phys. 7 (1974) 880

[19] B. Gravendeel, F. J. de Hoog and M. A. M. Schoenmakers, Fast photon counting in negative corona discharge in the Trichel regime, J. Phys. D: Appl. Phys. 21 (1988) 744

[20] A. A. Matveev and V. P. Silakov, Method of calculation of specific radiant emitting of the bands of 1 - and 2+ systems of nitrogen in the nonequilibrium nitrogen oxygen plasma, in Physics and Technology of Electric Power Transmission (MPEI, Moscow, 1998), pp. 201-218 (in Russian).

[21] A. F. Djakov, Yu. K. Bobrov, L. N. Bobrova, and Yu. V. Yourguelenas, Streamer discharge plasma parameters determination in air on a base of a measurement of radiation of the molecular bands of nitrogen, in Physics and Technology of Electric Power Transmission (MPEI, Moscow, 1998), pp 219-233 (in Russian).

[22] A. F. Djakov, Yu. K. Bobrov, and Yu. V. Yourguelenas, Modelling of a positive streamer in air in a non-uniform external field, in Physics and Technology of Electric Power Transmission (MPEI, Moscow, 1998), pp 161-200 (in Russian).

[23] Yu. V. Shcherbakov, Physical parameters of streamer in air, Internal Report of High Voltage Research Center No. 3237-B97. 45c Moscow Region, Russia, 1997 (in Russian).

[24] P. Paris, M. Aints, F. Valk, T. Plank, A. Haljaste, K. V. Kozlov and H.-E. Wagner, Intensity ratio of spectral bands of nitrogen as a measure of electric field strength in plasmas, J. Phys. D: Appl. Phys. 38 (2005) 3894

[25] Yu. V. Shcherbakov and R. S. Sigmond, Subnanosecond spectral diagnostics of streamer discharges: I. Basic experimental results, J. Phys. D: Appl. Phys. 40 (2007) 460-473

[26] Yu. V. Shcherbakov and R. S. Sigmond, Subnanosecond spectral diagnostics of streamer discharges: II. Theoretical background, J. Phys. D: Appl. Phys. 40 (2007) 474-487

[27] G. W. Trichel, The mechanism of the negative point to plane corona near onset, Phys. Rev. 54 (1938) 1078 
[28] H. Hoeft, M. Kettlitz, M. M. Becker, T. Hoder, D. Loffhagen, R. Brandenburg and K.-D. Weltmann, Breakdown characteristics in pulsed driven dielectric barrier discharges: influence of the prebreakdown phase due to volume memory effects, Journal of Physics D: Applied Physics, 46 (2014) 465206

[29] T. Hoder, Z. Bonaventura, A. Bourdon and M. Simek, Sub-nanosecond delays of light emitted by streamer in atmospheric pressure air: Analysis of $\mathrm{N}_{2}\left(\mathrm{C}^{3} \mathrm{Pi}\right)$ and $\mathrm{N}_{2}^{+}\left(\mathrm{B}^{2} \mathrm{Sigma}^{+}{ }_{u}\right)$ emissions and fundamental streamer structure, J. Appl. Phys. 117 (2015) 073302

[30] R. Brandenburg, T. Hoder, H.-E. Wagner, Two-dimensional spatially resolved cross-correlation spectroscopy of the microdischarge development in barrier discharges in air, IEEE Transactions on plasma science 36 (2008) 1318

[31] P. Kloc, H.-E. Wagner, D. Trunec, Z. Navratil and G. Fedoseev, An investigation of dielectric barrier discharge in Ar and Ar/NH3 mixture using cross-correlation spectroscopy, J. Phys. D: Appl. Phys. 43 (2010) 345205

[32] H. Grosch, T. Hoder, K.-D. Weltmann, and R. Brandenburg, Spatio-temporal development of microdischarges in a surface barrier discharge arrangement in air at atmospheric pressure, Eur. Phys. J. D 60 (2010) 547

[33] T. Hoder, M. Sira, K. V. Kozlov and H.-E. Wagner, Investigation of the coplanar barrier discharge in synthetic air at atmospheric pressure by cross-correlation spectroscopy, J. Phys. D: Appl. Phys. 41 (2008) 035212

[34] H. Hoeft, M. Kettlitz, K.-D. Weltmann and R. Brandenburg, The bidirectional character of $\mathrm{O}_{2}$ concentration in pulsed dielectric barrier discharges in $\mathrm{O}_{2} / \mathrm{N}_{2}$ gas mixtures, J. Phys. D: Appl. Phys. 47 (2014) 455202

[35] T. Gerling, T. Hoder, R. Brandenburg, R. Bussiahn and K.-D. Weltmann, Influence of the capillary on the ignition of the transient spark discharge, J. Phys. D: Appl. Phys. 46 (2013) 145205 (7pp)

[36] T. Gerling, T. Hoder, R. Bussiahn, R. Brandenburg and K.-D. Weltmann, On the spatio-temporal dynamics of a self-pulsed nanosecond transient spark discharge: a spectroscopic and electrical analysis, Plasma Sources Sci. Technol. 22 (2013) 065012 